\title{
Radio-pathological correlation of a retroperitoneal teratoma in a 2-year-old girl: a case report
}

\author{
Deki Choden ${ }^{1}$, Kinley Sangay Dorji², Sonam Choden ${ }^{3}$ \\ ${ }^{\prime}$ Department of Radio-diagnosis \& Imaging, Jigme Dorji Wangchuck National Referral Hospital, Thimphu, Bhutan \\ ${ }^{2}$ Department of Radio-diagnosis \& Imaging, Eastern Regional Referral Hospital, Mongar, Bhutan \\ ${ }^{3}$ Department of Pathology \& Laboratory Medicine, Jigme Dorji Wangchuck National Referral Hospital, Thimphu, Bhutan
}

\begin{abstract}
Retroperitoneal teratoma is a extragonadal germ cell tumor comprising 5\% of all teratomas in children, and the third most common retroperitoneal neoplasm in children. This is a case report of a 2 years old girl who presented with abdominal distension and palpable mass. The imaging findings of the mass was characteristic of retroperitoneal teratoma which was confirmed by histopathology report following complete excision. Retroperitoneal tumor is an uncommon tumor in children with characteristic imaging findings. Computed tomography is mainly used to evaluate the extent of the disease. Most of the retroperitoneal tumors are benign and curable with complete surgical excision.
\end{abstract}

Keywords: Germ cell tumor; Teratoma.

\section{INTRODUCTION}

Germ cell tumors (GCTs) are a heterogeneous group of tumors derived from one or more germ cell layers. It may arise from the gonads or extra-gonadal sites such as mediastinum, sacrococcygeal region, retroperitoneum and pineal gland ${ }^{1}$. The GCTs can be further subdivided into seminoma and nonseminomatous germ cell tumors. Teratoma is one of the subtypes of nonseminomatous germ cell tumor. Here, we report a case of retroperitoneal teratoma in a 2 years old girl who came with abdominal distention and palpable mass. The imaging findings were characteristic of retroperitoneal teratoma which was later confirmed by histopathology report following resection.

\section{CASE REPORT}

A 2 years old girl presented to Jigme Dorji Wangchuck National Referral Hospital in Bhutan with progressive abdominal distension for 2 months. She had no history of fever, loss of weight, bowel or bladder complaints. She demonstrated normal growth and

\section{Corresponding author:}

Kinley Sangay Dorji

kinleysangay1323@gmail.com development. On examination, the abdomen was distended with a large palpable firm mass in the epigastric and umbilical region. No tenderness or skin changes was observed. Routine blood tests were unremarkable. The serum alpha-fetoprotein (AFP) and betahuman chorionic gonadotropin $(\beta-\mathrm{HCG})$ were also within normal range measuring $1.57 \mathrm{ng} / \mathrm{mL}$ and $<0.100 \mathrm{mIU} / \mathrm{mL}$ respectively.

The ultrasound of abdomen revealed a large heterogeneous mass with multiple cystic components and internal echoes at epigastrium and left hypochondriac region [Figure 1]. Plain abdominal radiograph and computed tomography (CT) scan was advised for further evaluation of the mass.

Plain abdominal radiograph showed a radio-lucent mass in the epigastric region with a thick coarse internal calcification at T11 level. The mass was noted displacing right hepatic lobe and spleen laterally [Figure 2].
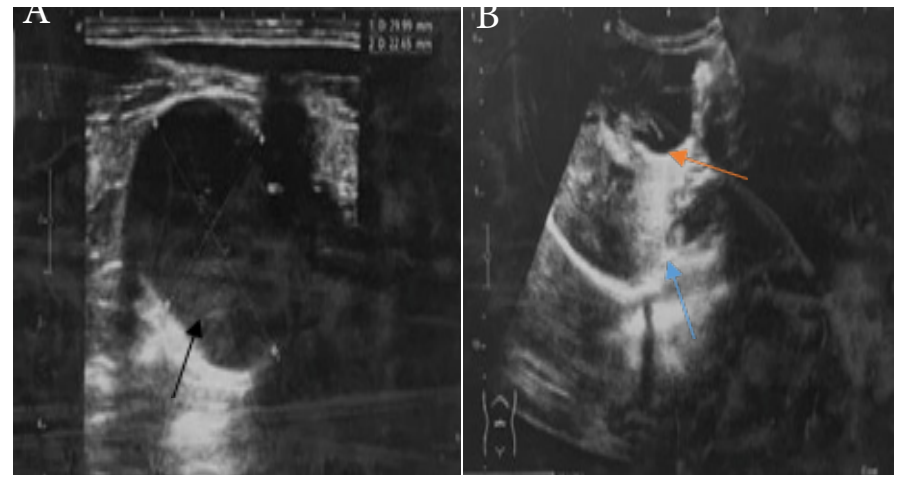

Figure 1. Ultrasound images of abdomen showing A) Hypoechoic mass (black arrow) and B) multiple cystic components (orange arrow) and internal echoes (blue arrow) 


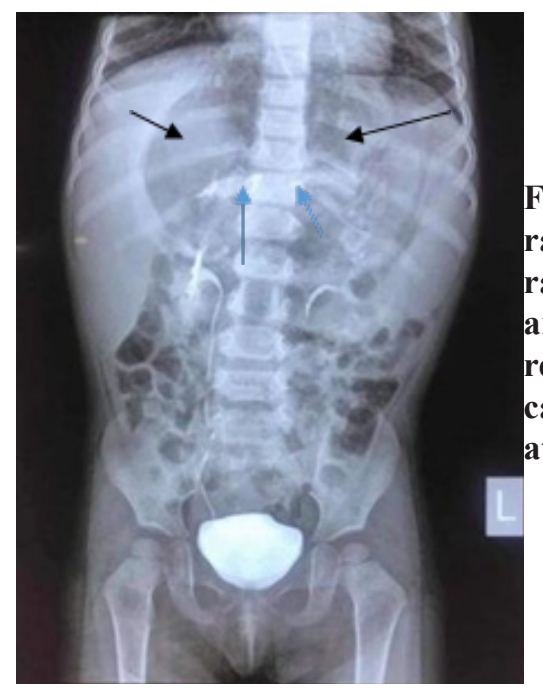

Figure 2. Plain abdominal radiograph showing a radio-lucent mass (black arrows) in epigastric region with a thick coarse calcification (blue arrows) at T11 level

Computed tomography (CT) scan of whole abdomen showed a large fairly well-defined mixed density mass, consisting of predominantly fatty component with multiple loculated cystic lesions, thick coarse calcifications and soft tissue components measuring about $9.0 \times 11.2 \mathrm{~cm}$ in the epigastric region. The mass was noted displacing the liver and spleen laterally, pancreas anterosuperiorly and bowel loops inferiorly [Figure 3]. No significant intra-abdominal nodes were found. The rest of the abdomen and pelvis were unremarkable.

a)
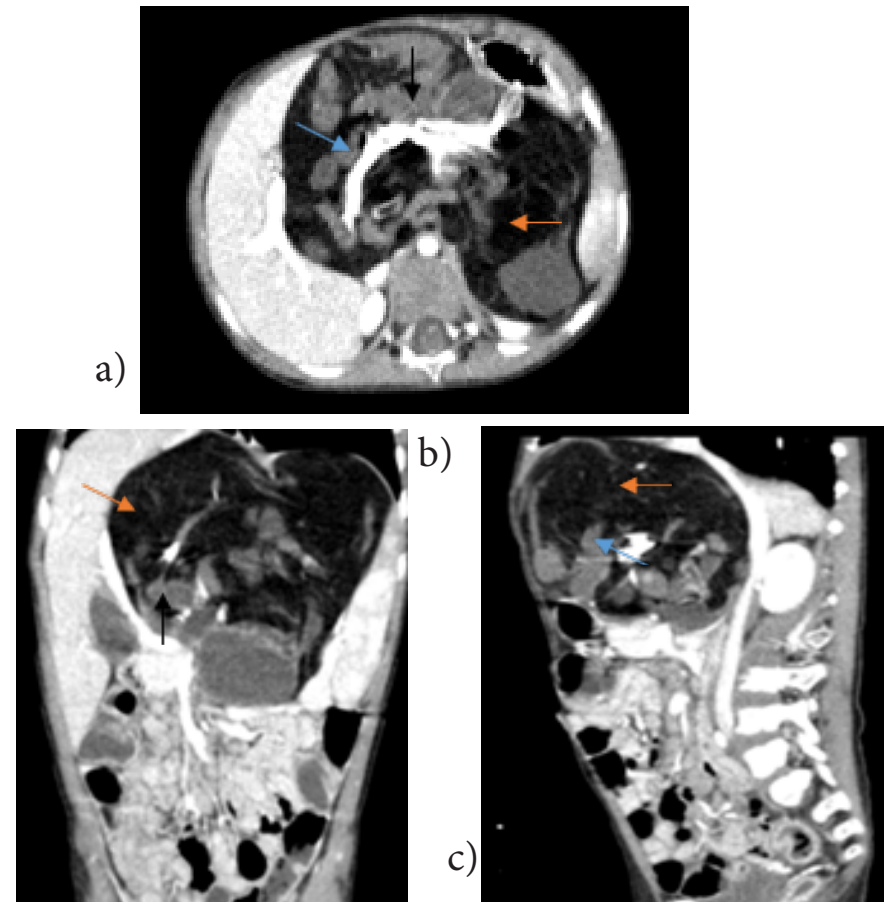

Figure 3. a) Axial, b) Coronal and c) Sagittal contrast enhanced images of whole abdomen reveals a large mixed density mass with fatty component (orange arrows), multiple loculated cysts, thick coarse calcification (blue arrows) and soft tissue components (black arrows) in the epigastric region
Exploratory laparotomy with tumor resection was performed for this patient. Intraoperative findings included a large multi-lobulated retroperitoneal mass measuring 10x12 cm in size. No major vessel encasement was found.

Grossly the mass was lobulated and encapsulated, measuring $12 \times 11 \times 5 \mathrm{~cm}$ (Figure 4 A \& B). Sections showed fibrofatty cut surfaces containing foci of red brown discoloration, cartilage, bone, small cystic lesions filled with sebaceous and mucoid material, and a focus of tan-white soft to rubbery lesion. The external surface was smooth and glistening. No capsular invasion was seen [Figure 4].
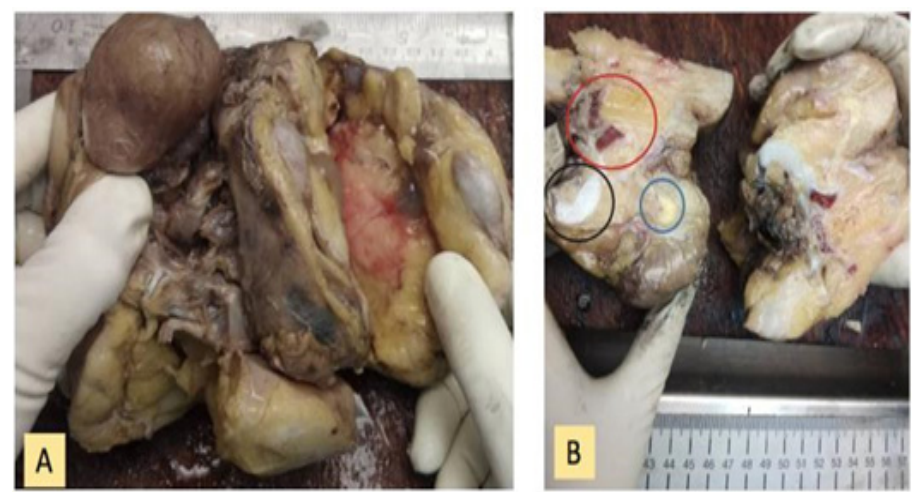

Figure 4. A \&B: Cartilage (black inset), bone with marrow (red inset), small cystic lesion filled with sebaceous material (blue inset)

Microscopically, the mass composed exclusively of mature tissue derived from all three germ cell layers (Figure 5). The ectodermal component consisted of skin with appendages, epidermal inclusion cyst and mature brain tissue. The mesodermal component consisted of islands of mature cartilage and bone tissue. The endodermal component consisted of bronchial and focal colonic tissue. The capsule was made up of thin layer of fibrotic tissue. No immature

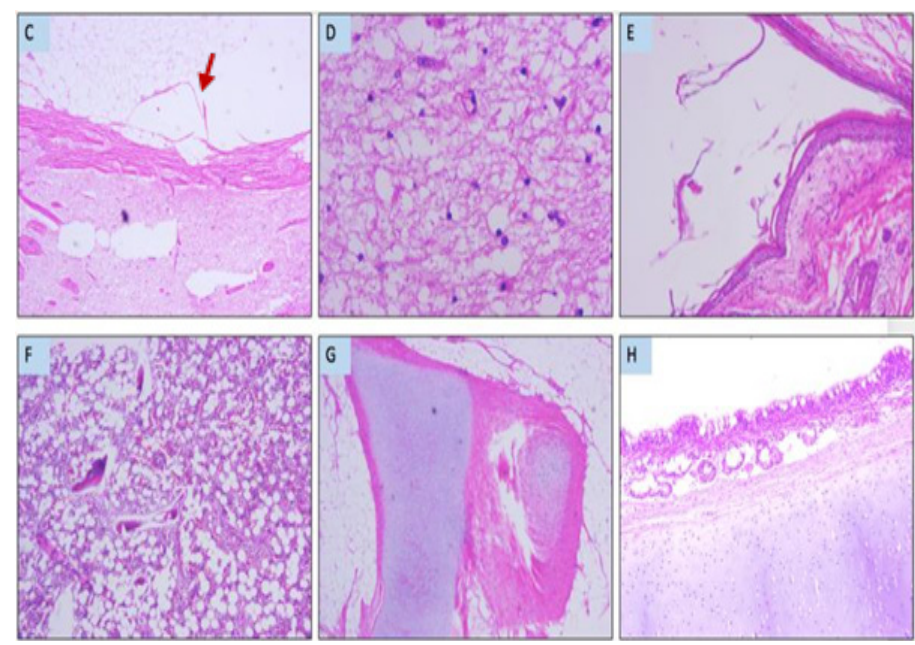

Figure 5. C. Mature adipose tissue (red arrow) with adjacent brain tissue (black arrow) (H\&E, 100x); D. Mature brain tissue (400x); E. Epidermal inclusion cyst (100x); F. Bone marrow (400x); G. Cartilage (400x); H. Bronchial tissue (400x) 
component or malignant transformation was seen. Thus, the final diagnosis was matured retroperitoneal teratoma was made.

The post-operative period was uneventful and she was discharged after 5 days.

\section{DISCUSSIONS}

Germ cell tumors (GCTs) are thought to occur due to abnormal differentiation of fetal germ cells that arises from the fetal yolk sac. If there is normal migration, it will give rise to gonadal germ cell tumors and if abnormal migration, it will be extragonadal germ cell tumors (EGGCTs). Around $30-40 \%$ of EGGCTs are seminomas and $60-70 \%$ are nonseminomatous germ cell tumors ${ }^{2}$. Nonseminomatous germ cell tumors can be further subdivided into yolk-sac tumors, embryonal carcinomas, choriocarcinomas, teratoma and non-teratomatous combined germ cell tumors.

Teratoma, by definition, include components derived from all three embryonic layers. Teratomas can either be mature or immature depending upon the presence of immature neuroectodermal elements. Among the many extra-gonadal sites, retroperitoneum is second commonest location after anterior mediastinum $^{2,3}$. Retroperitoneal teratoma comprise of $3.5-4 \%$ of germ cell tumors in children ${ }^{4}$. They mostly occur in children as seen our case report of a 2 years old girl which is compatible with prevalent epidemiology ${ }^{5}$.

Most common clinical presentation is abdominal distension and palpable mass. Our patient also presented with abdominal distension and palpable mass for 2 months duration. Usually, ultrasound is the first imaging modality for evaluation pediatric abdominal mass. However, the ultrasonographic findings of retroperitoneal teratoma are non-specific just like in our case. The plain abdominal radiograph is the next line of investigation. In retroperitoneal teratoma, plain abdominal X-ray may show calcification, like in our patient where there was a thick coarse calcification in the epigastric region of the abdomen, or formed bony components (considered pathognomonic). Infact Schey and Vesley suggested that presence of characteristic shard-like calcifications on plain abdominal X-ray should be adequate for tumor excision ${ }^{6}$. Despite all these evidences, CT is performed in most of the patients. The role of the $\mathrm{CT}$ includes making a diagnosis (especially when there is no or small calcification), to evaluate the extent of the disease and relationship to major vessels. CT findings of internal homogeneity, fat density, cyst formation and calcification are important predictor of benign retroperitoneal tumor. However, it has been found that CT tends to overestimate the tumor adherence to the adjacent structures when compared with real operative findings. Therefore, CT findings of retroperitoneal teratoma showing suspicious invasion of surrounding structures should not limit one from excision of the tumor. Among the blood report, serum alpha fetoprotein (AFP) is important as it can be raised in immature teratoma and in case of recurrence ${ }^{7-9}$. In our patient, CT image demonstrated well-defined mixed density mass consisting of fatty component, cysts and calcification suggestive of benign nature of the tumor. In addition, the serum AFP was also normal.

Complete excision of the tumor provides best chance of cure $^{7}$. Since most of the retroperitoneal teratoma are not malignant, non-mutilating excision is possible and should be attempted. Prognosis is generally good and curative after the complete tumor removal. Infact, one of the most important prognostic factor is the complete tumor removal as residual tumor might undergo malignant transformation. In our case, the patient underwent exploratory laparotomy with complete tumor excision. The histopathology report came out as mature retroperitoneal teratoma.

\section{CONCLUSIONS}

Retroperitoneal teratoma is an uncommon tumor in children with characteristic imaging findings. The presence of calcification, formed bone and teeth which can be seen in even in plain radiograph are pathognomic. CT is mainly used to evaluate the extent of the disease. Most of the retroperitoneal tumors are benign and curable with complete surgical excision. Prognosis is good and follow up is done with serum AFP.

\section{REFERENCES}

1. Faure-Conter C, Orbach D, Cropet C, Baranzelli MC, Martelli $H$, Thebaud E, et al. Salvage therapy for refractory or recurrent pediatric germ cell tumors: the French SFCE experience. Pediatr Blood Cancer. 2014 Feb 1; 61(2):253-9. [Full Text | DOI]

2. Extragonadal Germ Cell Tumors: Practice Essentials, Pathophysiology, Epidemiology. 2021 Aug 24. [Full Text]

3. Annigeri VM. Giant Retroperitoneal Mature Cystic Teratoma in an Infant: A Case Report. J Pediatr Neonatal Care. 2017 Dec 6; 5(7). [Full Text $\mid$ DOI]

4. Pan P. Retroperitoneal Teratoma in a Child with Clinical and Operative Photos. Austin J Surg. 2019; 6(3); 1165. [Full Text]

5. Khan SA, Mahmood T, Sarwar MZ, Rasool SH, Siddique MD, Khan Z. Retroperitoneal Teratoma in an adult presenting with painful abdominal mass: case history and literature review. 2012;5(1):3. [Full Text]

6. Schey WL, Vesely JJ, Radkowski MA. Shard-like calcifications in retroperitoneal teratomas. Pediatr Radiol. 1986; 16(1):824. [Full Text | DOI]

7. Rattan KN, Yadav H, Srivastava D, Rattan A. Childhood retroperitoneal teratomas: 5 years of experience in a tertiarycare hospital. Ann Pediatr Surg. 2016; 12(4):158-61.[Full Text $\mid$ DOI] 
8. Tripathy PK, Pattnaik K, Jena PK, Mohanty HK. Retroperitoneal Mature Cystic Teratoma in a 3-month-old Infant: A Case Report and Literature Review. Indian J Med Paediatr Oncol. 2018 Jan; 39(01):117-9. [Full Text]
9. Singh C, Raypattanaik NM, Sharma I, Kaman L. Primary Retroperitoneal Teratoma in a Young Male: A Case Report. Cureus. 2021 Jun 1; 13(6). [Full Text | DOI] 